\title{
Fish Diversity of Coastal Andhra Pradesh, Southeast Coast of India
}

\section{Darwin Chatla, P. Padmavathi*}

\author{
Department of Zoology and Aquaculture, Acharya Nagarjuna University, Nagarjuna Nagar - 522 510, Andhra
} Pradesh, India.

\begin{abstract}
Diversity of marine fishes was studied along the coast of Bay of Bengal from four selected landing stations of Andhra Pradesh, Southeast coast of India during the period from December 2016 to November 2018. A total of 171 species belonging to 14 orders, 63 families and 128 genera have been recorded during the study period. It is evident that marine fish production is well below the production targets. The less availability of some species indicates a remarkable decline in the diversity of fishes. The anthropogenic disturbances and climatic changes are reported to be the factors affecting the fish population and diversity. Therefore, we are in the stage of need of the hour to conserve marine biodiversity in coastal Andhra Pradesh. The current study also recorded the IUCN status of 171 fish species in various categories of conservation status.

Keywords | Bio-diversity, Conservation, IUCN status, Marine fisheries, Andhra Pradesh

Received | March 10, 2021; Accepted | April 26, 2021; Published | July 28, 2021

${ }^{*}$ Correspondence | P. Padmavathi, Department of Zoology and Aquaculture, Acharya Nagarjuna University, Nagarjuna Nagar - 522 510, Andhra Pradesh, India; Email: padmapin@yahoo.com

Citation | Chatla D, Padmavathi P (2021). Fish diversity of coastal Andhra Pradesh, southeast coast of India. Adv. Anim. Vet. Sci. 9(9): 1424-1436.

DOI | http://dx.doi.org/10.17582/journal.aavs/2021/9.9.1424.1436

ISSN (Online) | 2307-8316; ISSN (Print) | 2309-3331
\end{abstract}

Copyright $\odot 2021$ Chatla and Padmavathi. This is an open access article distributed under the Creative Commons Attribution License, which permits unrestricted use, distribution, and reproduction in any medium, provided the original work is properly cited.

\section{INTRODUCTION}

$\mathrm{I}^{\mathrm{s}}$ ndia is one of the largest mega-diversity countries and known for its biodiversity richness abundance reflected in the diversity of fresh and marine water fishes. It is wellknown that the marine faunal diversity depends mostly on fish diversity (Kar et al., 2017). It was estimated that India housed 3231 valid species, of which 2443 are marine (75.6\%) (Gopi and Mishra, 2015). Globally, India occupies the second-largest fish producer with 3.56 million tonnes for the year 2019 (FRAD, 2019) and exports worth US $\$ 7.08$ billion (DADF, 2019). The fisheries play a remarkable role in the agro-economy of India with regard to protein supply and employment (Chatla et al., 2020).

The state of Andhra Pradesh endowed with a vast coastline of $974 \mathrm{~km}$ spread across nine coastal districts is situated between $15^{\circ} 54^{\prime} 46.44^{\prime \prime} \mathrm{N}$ latitude and $79^{\circ} 44^{\prime} 23.95^{\prime \prime}$ E longitude. It is scattered with 353 fish landing stations including 4 major harbors viz., Visakhapatnam, Kakinada, Machilipatanam, and Nizampatnam (CMFRI, 2012).
Andhra Pradesh has known for its healthy fishing grounds and diverse resources comprising different gears and crafts with a total number of 27211 fishing vessels, including 1675 mechanized, 11807 motorized and 13729 traditional fishing crafts, and gears such as seines, cast nets, drag nets, gill nets, trawl nets, and hook and lines (Rao et al., 2008; WAPCOS, 2017). The marine fish landings accounted for about 0.259 million tonnes (mt) in 2019 (FRAD, 2020). However, fish landings have fluctuated over the years from 2013 to 2019 (Figure 1).

Every region of the sea is a home for wide variety of life and repository of biodiversity. Among marine biodiversity, fish diversity is comparatively higher than other faunal diversity with ample existing data and higher probability for the discovery of new species (Pyle et al., 2019). The dispersion patterns of several species and extinction of indigenous fishes have been directly linked to human interference (Nelson et al., 2016). Of late, over exploitation of fish species has become a matter of great concern (Ranjan, 2018). The distribution of fishery resources in the 
coastal waters varies with distance from the shore (Vardharajan and Soundarapandian, 2015). The catchment rates vary with the landing stations and the species (Kar et al., 2017), and hence the catchment information is essential for the development of effective management and to develop the measures to be taken to conserve the fishes towards sustainable utilization (Darwin and Padmavathi, 2020). In view of the given significance of diversity, the fishery composition of landings and seasonal abundance of different resources along the Visakhapatnam coast were studied by Sudarsan (1981), Krishnan and Mishra (1993), Sujatha (1995), Barman et al. (2004) and Sreedhar et al. (2009). However, there is no documented evidence on the diversity of fishes in other fish landing stations of coastal Andhra Pradesh. Therefore, the current study has been undertaken to know the marine fish diversity of unexplored four fish landing stations of coastal Andhra Pradesh.

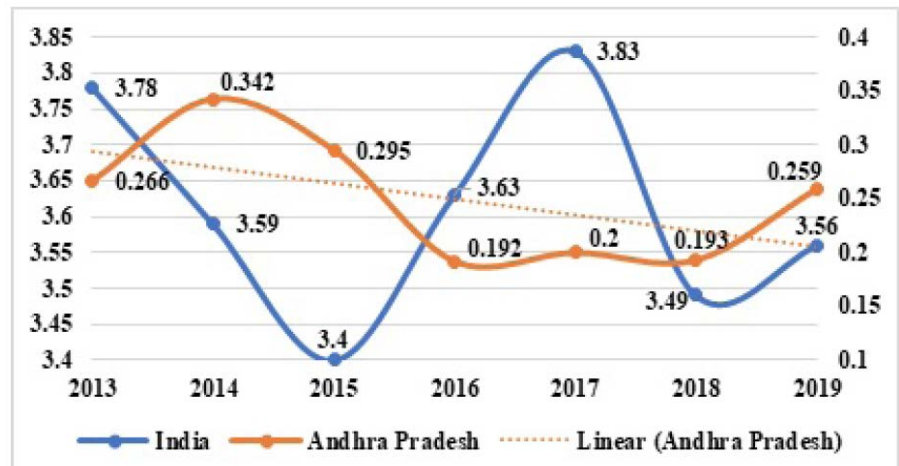

Figure 1: Annual Marine Fish Landings in Andhra Pradesh and India (Source CMFRI).

\section{MATERIALS AND METHODS}

\section{STUdy Area}

The present study is based on the samples collected in four designated landing stations of coastal Andhra Pradesh viz., Machilipatnam (A), Nizampatnam (B), Vodarevu (C), and Pakala (D) (Figure 2) located in Krishna, Guntur, and Prakasam districts along $224 \mathrm{~km}$ coastline (Table 1 and 2).

\section{Sample Collection}

To study the marine fish diversity, fish samples were collected from the catches of fish landing centres where fishes were caught by using different gears such as cast nets, trammel net, hook and line, shore seine, boat seine and purse seine. Sampling was done at regular fortnight or monthly intervals in all four landing stations from December 2016 to November 2018 except fish ban period of the months of April and May. The fish samples from various catches were collected as soon as the catches were offloaded. Some of the samples were preserved in 5\% formalin and transported to the laboratory for taxonomic identification. All the collections after identification were documented at Museum, Department of Zoology and Aquaculture, Acharya Nagar- juna University, Andhra Pradesh, India.
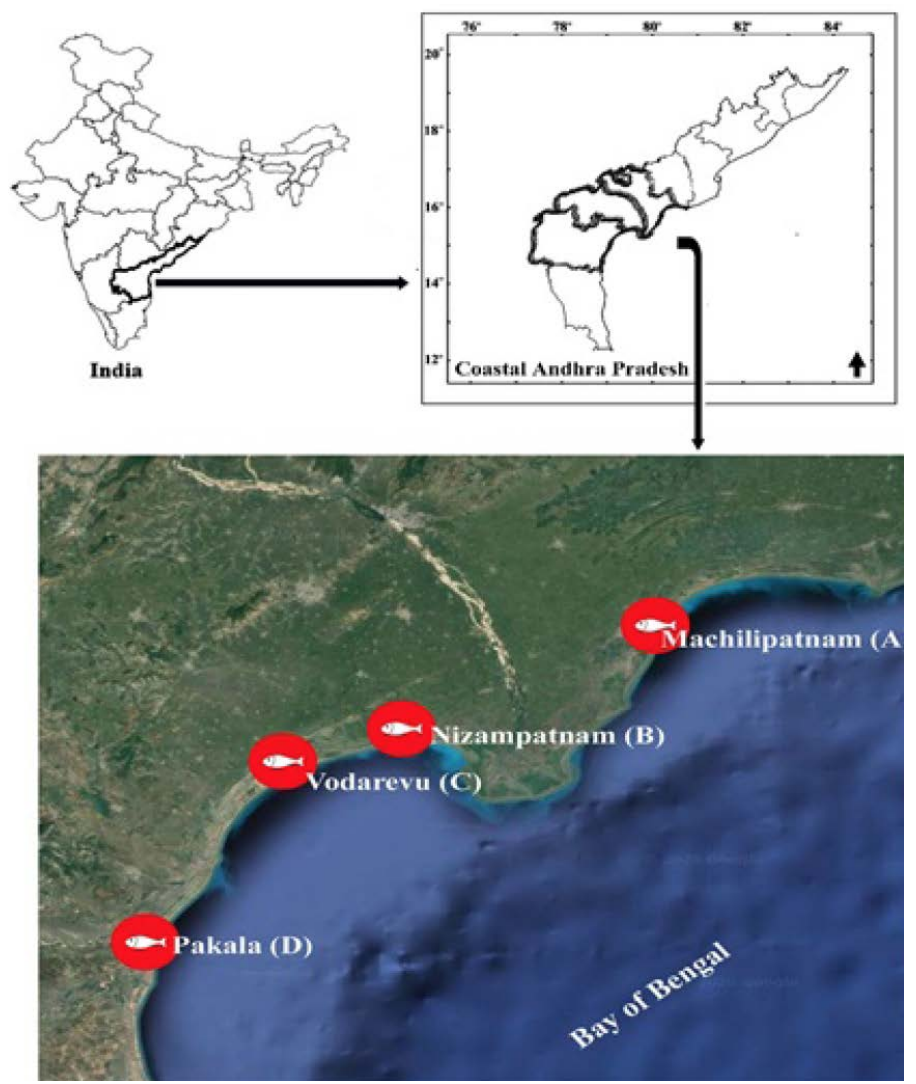

Figure 2: Geographical location of the four fish landing stations.

Table 1: Geographical location of the four sampling sites of coastal Andhra Pradesh.

$\begin{array}{lll}\text { Fish landing stations } & \begin{array}{l}\text { Geographical location of fish landing } \\ \text { stations }\end{array} \\ & \text { Latitude (North) } & \text { Longitude (East) } \\ \text { Machilipatnam (A) } & 1^{\circ} 14^{\prime} 49^{\prime \prime} & 81^{\circ} 18^{\prime} 63^{\prime \prime} \\ \text { Nizampatnam (B) } & 15^{\circ} 52^{\prime} 58^{\prime \prime} & 80^{\circ} 38^{\prime} 18^{\prime \prime} \\ \text { Vodarevu (C) } & 15^{\circ} 79^{\prime} 34^{\prime \prime} & 80^{\circ} 41^{\prime} 10^{\prime \prime} \\ \text { Pakala (D) } & 15^{\circ} 27^{\prime} 31^{\prime \prime} & 80^{\circ} 08^{\prime} 53^{\prime \prime}\end{array}$

\section{Fish Diversity and Abundance}

Fishes were identified up to the species level by following the standard books (Talwar and Kacker, 1984; Barman et al., 2004) and web-based keys, FishBase (www.fishbase.in) (Froese and Pauly, 2020), and Eschmeyer's Catalog of Fishes (www.calacademy.org) (Fricke et al., 2020). The classification adopted was mainly followed by Nelson (2006). The current valid names, common names and concise data on the conservation status of fish species and importance to fisheries was gathered based on the FishBase (Froese and Pauly, 2020), International Union for Conservation of Nature (www.iucn.org) (IUCN, 2019) and World Register of Marine Species (www.marinespecies.org) (WoRMS, 2020). The percentage composition of orders, families, genera and species of collected fishes was recorded. 
Table 2: fish species, Common names, Threat to humans, IUCN status and occurrence stations in coastal Andhra Pradesh.

\section{Class: Chondrichthyes/Elasmobranchii}

\begin{tabular}{|c|c|c|c|c|c|c|c|c|}
\hline $\begin{array}{l}S \text {. } \\
\text { No. }\end{array}$ & Order & Family & Species Name & $\begin{array}{l}\text { Common } \\
\text { Name }\end{array}$ & Station & $\begin{array}{l}\text { IUCN } \\
\text { Status }\end{array}$ & $\begin{array}{l}\text { Threat } \\
\text { to } \mathrm{Hu}- \\
\text { mans }\end{array}$ & $\begin{array}{l}\mathrm{Hu}- \\
\text { man } \\
\text { Use }\end{array}$ \\
\hline 1 & Carcharhiniformes & Carcharhinidae & $\begin{array}{l}\text { Carcharbinus dussumie- } \\
\text { ri (Müller \& Henle, 1839) }\end{array}$ & $\begin{array}{l}\text { White cheek } \\
\text { shark }\end{array}$ & $A, B$ & $\mathrm{EN}$ & $\mathrm{H}$ & $\mathrm{C}$ \\
\hline 2 & & & C. limbatus (Müller \& Henle, 1839) & Blacktip shark & $\mathrm{A}, \mathrm{B}, \mathrm{C}$ & VU & $\operatorname{Tr}$ & $\mathrm{C}, \mathrm{Gf}$ \\
\hline 3 & & & C. sorrah (Müller \& Henle, 1839) & Spot-tail shark & $\mathrm{A}, \mathrm{B}, \mathrm{C}$ & NT & $\mathrm{H}$ & $\mathrm{C}$ \\
\hline 4 & & & $\begin{array}{l}\text { Lamiopsis temminck- } \\
\text { ii (Müller \& Henle, 1839) }\end{array}$ & Broadfin Shark & $\mathrm{A}, \mathrm{B}$ & EN & $\mathrm{H}$ & $\mathrm{C}$ \\
\hline 5 & & & Rhizoprionodon acutus (Rüppell, 1837) & Milk shark & $\mathrm{A}, \mathrm{B}, \mathrm{C}$ & $\mathrm{LC}$ & $\mathrm{H}$ & $\mathrm{C}$ \\
\hline 6 & & & $\begin{array}{l}\text { Scoliodon laticaudus Müller } \\
\& \text { Henle, } 1838\end{array}$ & $\begin{array}{l}\text { Spadeno- } \\
\text { se shark }\end{array}$ & $\mathrm{A}, \mathrm{B}$ & NT & $\mathrm{H}$ & $\mathrm{C}$ \\
\hline 7 & & Sphyrnidae & Sphyrna zygaena (Linnaeus, 1758) & $\begin{array}{l}\text { Hammer } \\
\text { head shark }\end{array}$ & $\begin{array}{l}\text { A, B } \\
\text { C, D }\end{array}$ & VU & $\operatorname{Tr}$ & $\mathrm{C}, \mathrm{Gf}$ \\
\hline 8 & Myliobatiformes & Aetobatidae & $\begin{array}{l}\text { Aetobatus flagellum (Bloch } \\
\& \text { Schneider, 1801) }\end{array}$ & $\begin{array}{l}\text { Longheaded } \\
\text { eagle ray }\end{array}$ & $\mathrm{A}, \mathrm{B}$ & $\mathrm{EN}$ & $\mathrm{H}$ & $\mathrm{C}$ \\
\hline 9 & & Dasyatidae & Himantura fava (Annandale, 1909) & $\begin{array}{l}\text { Honeycomb } \\
\text { whipray }\end{array}$ & $A, B$ & $\mathrm{NE}$ & $\mathrm{H}$ & Ss \\
\hline 10 & & & H. uarnak (Gmelin, 1789) & $\begin{array}{l}\text { Honeycomb } \\
\text { stingray }\end{array}$ & $\mathrm{A}, \mathrm{B}$ & VU & $\operatorname{Tr}$ & $\mathrm{C}, \mathrm{Gf}$ \\
\hline 11 & & & Maculabatis gerrardi (Gray, 1851) & $\begin{array}{l}\text { Sharp nose } \\
\text { stingray }\end{array}$ & $\begin{array}{l}\mathrm{A}, \mathrm{B} \\
\mathrm{C}, \mathrm{D}\end{array}$ & VU & $\mathrm{H}$ & $\mathrm{C}, \mathrm{Gf}$ \\
\hline 12 & & & $\begin{array}{l}\text { Telatrygon zugei (Müller } \\
\text { \& Henle, 1841) }\end{array}$ & $\begin{array}{l}\text { Pale-edged } \\
\text { stingray }\end{array}$ & $A, B$ & NT & $\mathrm{H}$ & $\mathrm{C}$ \\
\hline 13 & Orectolobiformes & Hemiscylliidae & $\begin{array}{l}\text { Chiloscyllium griseum } \\
\text { Müller \& Henle, } 1838\end{array}$ & $\begin{array}{l}\text { Grey bam- } \\
\text { booshark }\end{array}$ & $\begin{array}{l}\mathrm{A}, \mathrm{B} \\
\mathrm{C}, \mathrm{D}\end{array}$ & NT & $\mathrm{H}$ & $\mathrm{C}$ \\
\hline 14 & Rhinopristiformes & Rhinidae & $\begin{array}{l}\text { Rhynchobatus djidden- } \\
\text { sis (Forsskål, 1775) }\end{array}$ & Giant guitarfish & $\mathrm{A}, \mathrm{B}$ & VU & $\mathrm{H}$ & $\mathrm{C}, \mathrm{Gf}$ \\
\hline 15 & & Rhinobatidae & Rbinobatos annandalei Norman, 1926 & $\begin{array}{l}\text { Annandale's } \\
\text { guitarfish }\end{array}$ & B & $\mathrm{DD}$ & $\mathrm{H}$ & $\mathrm{F}$ \\
\hline 16 & Torpediniformes & Narcinidae & Narcine brunnea Annandale, 1909 & $\begin{array}{l}\text { Brown elec- } \\
\text { tric ray }\end{array}$ & $\mathrm{A}, \mathrm{B}$ & $\mathrm{NE}$ & $\mathrm{H}$ & Ss \\
\hline 17 & & Narkidae & $\begin{array}{l}\text { Narke dipterygia (Bloch } \\
\& \text { Schneider, 1801) }\end{array}$ & Numbray & $A, B$ & $\mathrm{DD}$ & $\mathrm{H}$ & $\mathrm{C}$ \\
\hline
\end{tabular}

\section{Class: Osteichthyes/Actinopterygii}

\begin{tabular}{|c|c|c|c|c|c|c|c|c|}
\hline 18 & Anguilliformes & Muraenidae & Strophidon sathete (Hamilton, 1822) & $\begin{array}{l}\text { Slender gi- } \\
\text { ant moray }\end{array}$ & B & $\mathrm{NE}$ & $\operatorname{Tr}$ & $\mathrm{C}, \mathrm{Gf}$ \\
\hline 19 & & $\begin{array}{l}\text { Muraenesoci- } \\
\text { dae }\end{array}$ & Muraenesox bagio (Hamilton, 1822) & $\begin{array}{l}\text { Common } \\
\text { pike conger }\end{array}$ & A, B & $\mathrm{NE}$ & $\mathrm{H}$ & $\mathrm{C}, \mathrm{Gf}$ \\
\hline 20 & & & Congresox talabonoides (Bleeker, 1853) & $\begin{array}{l}\text { Indian pike } \\
\text { conger }\end{array}$ & $\begin{array}{l}\mathrm{A}, \mathrm{B} \\
\mathrm{C}, \mathrm{D}\end{array}$ & NE & $\mathrm{H}$ & $\mathrm{C}$ \\
\hline 21 & & Ophichthidae & $\begin{array}{l}\text { Lamnostoma orientalis (Mc- } \\
\text { Clelland, 1844) }\end{array}$ & $\begin{array}{l}\text { Oriental } \\
\text { worm-eel }\end{array}$ & $\mathrm{B}, \mathrm{C}$ & $\mathrm{LC}$ & $\mathrm{H}$ & $\mathrm{C}$ \\
\hline 22 & Aulopiformes & Synodontidae & Harpadon nehereus (Hamilton, 1822) & Bombay duck & $\begin{array}{l}\mathrm{A}, \mathrm{B} \\
\mathrm{C}, \mathrm{D}\end{array}$ & $\mathrm{NE}$ & $\mathrm{H}$ & $\mathrm{C}$ \\
\hline 23 & & & Saurida tumbil (Bloch, 1795) & $\begin{array}{l}\text { Greater liz- } \\
\text { ardfish }\end{array}$ & $\begin{array}{l}\text { A, B, } \\
\text { C, D }\end{array}$ & $\mathrm{LC}$ & $\mathrm{H}$ & $\mathrm{C}$ \\
\hline 24 & Beloniformes & Belonidae & Xenentodon cancila (Hamilton, 1822) & Needle fish & $\mathrm{C}, \mathrm{D}$ & $\mathrm{LC}$ & $\operatorname{Tr}$ & $\mathrm{C}, \mathrm{Aq}$ \\
\hline 25 & & & $\begin{array}{l}\text { Strongylura strongylu- } \\
\text { ra (vanHasselt, 1823) }\end{array}$ & $\begin{array}{l}\text { Spottail } \\
\text { needlefish }\end{array}$ & $\begin{array}{l}\mathrm{A}, \mathrm{B} \\
\mathrm{C}, \mathrm{D}\end{array}$ & $\mathrm{NE}$ & $\mathrm{H}$ & $\mathrm{C}, \mathrm{Gf}$ \\
\hline
\end{tabular}

September 2021 | Volume 9 | Issue 9 | Page 1426 
26

27

28 Clupeiformes

29

30

31

32

33

34

35

36

37

38

39

40

41

42

43

44

45

46

47

48

49

50 Elopiformes

51 Mugiliformes

Elopidae

Mugilidae

Clupeidae

Engraulidae

C. nudus Swainson, 1839

Hilsa kelee (Cuvier, 1829)

Sardinella brachysoma Bleeker, 1852

S. fimbriata (Valenciennes, 1847)

S. gibbosa (Bleeker, 1849)

S. longiceps Valenciennes, 1847

Tenualosa ilisha (Hamilton, 1822)

Coilia dussumieri Valenciennes, 1848

Goldspot-

ted grenadier

anchovy

Encrasicholina devisi (Whitley, 1940)

Devis' anchovy

A, B

Commerson's A, B, LC H C

anchovy

C, D

nii Lacepède, 1803

Indian anchovy

A, B

Thryssa dussumieri (Va-

lenciennes, 1848)

T. hamiltonii Gray, 1835

T. malabarica (Bloch, 1795)

T. mystax (Bloch \& Schneider, 1801)

T. setirostris (Broussonet, 1782)

Pristigasteridae Amblygaster leiogaster (Valenciennes, 1847)

Anodontostoma chacun-

da (Hamilton, 1822)

Ilisha melastoma (Bloch

\& Schneider, 1801)

Nematalosa nasus (Bloch, 1795 )

Opisthopterus tardoore (Cuvier, 1829)

Dussumier's thryssa

A, B, LC H C

Hamilton's

C, D thryssa

Malabar thryssa A, B, DD H C

C, D

Moustached A, B

thryssa C, D

Longjaw thryssa A, B, LC H C

$\mathrm{C}, \mathrm{D}$

Smoothbelly B, C, D LC H F

sardinella

Chacunda A, B, LC H

gizzard shad

C, D

Indian ilisha

A, B LC H C

Bloch's giz- $\quad$ A, B, LC $\quad \mathrm{H} \quad \mathrm{C}$ zard shad C, D

Elops machnata (Forsskål, 1775) Tenpounder $\quad$ A, B, D LC $\quad H \quad$ C, Gf

Chelon parsia (Hamilton, 1822)

Goldspot mullet A, B, NE H C

C, D

C. planiceps (Valenciennes, 1836) Tade gray mullet B, C NE $\quad \mathrm{H} \quad \mathrm{C}$ 
53

54

55 Perciformes

56

57

58

59

60

61

62

63

64

65

66

67

68

69

70

71

72

73

74

75

76

77

78

79

80

69

Mugil cephalus Linnaeus, 1758

Planiliza macrolepis (Smith, 1846)

Acanthuridae Acanthurus mata (Cuvier, 1829)

Ambassidae

Ambassis nalua (Hamilton, 1822)

Chanda nama Hamilton, 1822

Apogonidae

Fibramia lateralis (Va-

lenciennes, 1832)

Carangidae

Atropus atropos (Bloch \&

Schneider, 1801)

Alectis indica (Rüppell, 1830)

Carangoides malabaricus (Bloch

\& Schneider, 1801)

Caranx ignobilis (Forsskål, 1775)

C. sexfasciatus Quoy \&

Gaimard, 1825

Decapterus russelli (Rüppell, 1830) Indian scad

Parastromateus niger (Bloch, 1795)

Selar crumenophthalmus (Bloch, 1793) Bigeye scad

Trachinotus blochii (Lacepède, 1801)

Cynoglossidae

Cynoglossus bilineatus (La-

cepède, 1802)

C. cynoglossus (Hamilton, 1822)

C. macrolepidotus (Bleeker, 1851)

C. semifasciatus Day, 1877

Paraplagusia bilineata (Bloch, 1787)

Derepanidae

Drepane punctata (Linnaeus, 1758)

Ephippidae

Gerridae

Ephippus orbis (Bloch, 1787)

Gerres filamentosus Cuvier, 1829

G. setifer (Hamilton, 1822)

Gobiidae

Boleophthalmus dussumie-

ri Valenciennes, 1837

P. novemradiatus (Hamilton, 1822)

Haemulidae

Pentaprion longimanus (Cantor, 1849)

Pomadasys maculatus (Bloch, 1793)
Flathead grey mullet

Largescale mullet

Elongate surgeonfish

$\begin{array}{lllll}\begin{array}{l}\text { Scalloped } \\ \text { perchlet }\end{array} & \text { A, B } & \text { LC } & \text { H } & \text { Ukn }\end{array}$

Elongate glass-perchlet

Humpback C, D LC H Ss

cardinal

Cleft bel- A, B, LC H $\quad$ C

ly trevally

Indian
threadfish

Malabar trevally A, B LC $\quad$ H $\quad$ C, Gf

Giant trevally A, B LC Pn $\quad$ C, Gf

Bigeye trevally A LC H C

Indianscad $\quad A, B, \quad$ LC $\quad H \quad$ C

$\mathrm{C}, \mathrm{D}$

Black pomfret

$\mathrm{A}, \mathrm{B}, \quad \mathrm{LC} \quad \mathrm{H} \quad \mathrm{C}$

C, D

A, B LC Pn C, Gf

Indian pompano A LC Pn $\quad$, Gf

Fourlined A, B NE $\mathrm{H} \quad \mathrm{C}$

tonguesole

Bengal A, B, NE H $\quad$ C

tongue sole

C, D

Large scale tongue sole

A, B NE H Ss

Bengal A, B NE H Ss

tonguesole

Doublelined A, B, NE $\mathrm{H} \quad \mathrm{C}$ tonguesole $\quad \mathrm{C}, \mathrm{D}$

Spotted A, B, NE H $\quad$ C, Aq sicklefish $\quad$ C, D

Orbfish $\quad \mathrm{B} \quad \mathrm{NE} \quad \mathrm{H} \quad \mathrm{C}$

Whipfin B, C, D LC H C silver-biddy

Small Bengal A, B NE H C silverbiddy

Mudskipper $\quad$ C, D LC $\quad H \quad$ Ukn

Pearse's mud- $\quad$ B, C, D DD $\quad \mathrm{H} \quad$ Ukn skipper

Longfin mojarra A, B LC $\mathrm{H} \quad \mathrm{C}$ Saddle grunt A LC $\mathrm{H} \quad \mathrm{C}$ 
Istiophoridae Istiophorus platypterus (Shaw, 1792) Indo-Pacif- $\quad$ A, B, $\quad$ LC $\quad$ H $\quad$ C, Gf ic sailfish

C, D

\begin{tabular}{|c|c|c|c|c|c|c|c|}
\hline 82 & & Istiompax indica (Cuvier, 1832) & Black marlin & A, B & DD & $\mathrm{H}$ & $\mathrm{C}, \mathrm{Gf}$ \\
\hline 83 & Lactariidae & $\begin{array}{l}\text { Lactarius lactarius (Bloch } \\
\& \text { Schneider, 1801) }\end{array}$ & False trevally & A, B & $\mathrm{NE}$ & $\mathrm{H}$ & $\mathrm{C}$ \\
\hline 84 & Latidae & Lates calcalifer (Bloch, 1790) & Barramundi & $\begin{array}{l}\mathrm{A}, \mathrm{B} \\
\mathrm{C}, \mathrm{D}\end{array}$ & $\mathrm{NE}$ & $\mathrm{H}$ & $\mathrm{C}, \mathrm{Ac}$ \\
\hline 85 & Leiognathidae & Eubleekeria splendens (Cuvier, 1829) & $\begin{array}{l}\text { Splendid } \\
\text { ponyfish }\end{array}$ & $\mathrm{A}, \mathrm{B}$ & $\mathrm{LC}$ & $\mathrm{H}$ & $\mathrm{C}$ \\
\hline 86 & & Leiognathus equulus (Forsskål, 1775) & $\begin{array}{l}\text { Common } \\
\text { ponyfish }\end{array}$ & $\begin{array}{l}\mathrm{A}, \mathrm{B} \\
\mathrm{C}, \mathrm{D}\end{array}$ & $\mathrm{LC}$ & $\mathrm{H}$ & $\mathrm{C}$ \\
\hline 87 & & $\begin{array}{l}\text { Karalla dussumieri (Va- } \\
\text { lenciennes, 1835) }\end{array}$ & $\begin{array}{l}\text { Dussumier's } \\
\text { ponyfish }\end{array}$ & $A, B$ & $\mathrm{NE}$ & $\mathrm{H}$ & $\mathrm{C}$ \\
\hline 88 & & Nuchequula gerreoides (Bleeker, 1851) & $\begin{array}{l}\text { Decorated } \\
\text { ponyfish }\end{array}$ & B & $\mathrm{NE}$ & $\mathrm{H}$ & Ss \\
\hline 89 & & $\begin{array}{l}\text { Photopectoralis bindus (Va- } \\
\text { lenciennes, 1835) }\end{array}$ & $\begin{array}{l}\text { Orangefin } \\
\text { ponyfish }\end{array}$ & A, B & $\mathrm{NE}$ & $\mathrm{H}$ & $\mathrm{C}$ \\
\hline 90 & & Secutor insidiator (Bloch, 1787) & $\begin{array}{l}\text { Pugnose } \\
\text { ponyfish }\end{array}$ & A, B & $\mathrm{NE}$ & $\mathrm{H}$ & $\mathrm{C}$ \\
\hline 91 & & S. ruconius (Hamilton, 1822) & $\begin{array}{l}\text { Deep pugnose } \\
\text { ponyfish }\end{array}$ & $A, B$ & NE & $\mathrm{H}$ & $\mathrm{C}$ \\
\hline 92 & Lobotidae & Lobotes surinamensis (Bloch, 1790) & Tripletail & $A, B$ & $\mathrm{LC}$ & $\mathrm{H}$ & $\mathrm{C}, \mathrm{Gf}$ \\
\hline 93 & Lutjanidae & $\begin{array}{l}\text { Lutjanus argentimacula- } \\
\text { tus (Forsskål, 1775) }\end{array}$ & $\begin{array}{l}\text { Mangrove } \\
\text { red snapper }\end{array}$ & A & $\mathrm{LC}$ & Pn & $\mathrm{C}, \mathrm{Gf}$ \\
\hline 94 & & $\begin{array}{l}\text { L. indicus Allen, White } \\
\text { \& Erdmann, } 2013\end{array}$ & Snapper fish & A, B & $\mathrm{LC}$ & $\mathrm{H}$ & Ss \\
\hline 95 & & L. johnii (Bloch, 1792) & John's snapper & $\begin{array}{l}\text { A, B, } \\
\text { C, D }\end{array}$ & NE & $\mathrm{H}$ & $\mathrm{C}, \mathrm{Gf}$ \\
\hline
\end{tabular}

\begin{tabular}{|c|c|c|c|c|c|c|c|}
\hline 96 & & L. russellii (Bleeker, 1849) & Russell's snapper & $A, B$ & NE & $\mathrm{H}$ & $\mathrm{C}$ \\
\hline 97 & Menidae & $\begin{array}{l}\text { Mene maculate (Bloch \& } \\
\text { Schneider, 1801) }\end{array}$ & Moonfish & $\mathrm{A}, \mathrm{B}$ & $\mathrm{NE}$ & $\mathrm{H}$ & $\mathrm{C}$ \\
\hline 98 & Mullidae & Parupeneus indicus (Shaw, 1803) & Indian goatfish & A, B & $\mathrm{LC}$ & $\mathrm{H}$ & $\mathrm{C}, \mathrm{Gf}$ \\
\hline 99 & & Upeneus vittatus (Forsskål, 1775) & $\begin{array}{l}\text { Yellow striped } \\
\text { goatfish }\end{array}$ & $\begin{array}{l}\text { A, B, } \\
\text { C, D }\end{array}$ & $\mathrm{LC}$ & $\mathrm{H}$ & $\mathrm{C}, \mathrm{Aq}$ \\
\hline 100 & Nemipteridae & Nemipterus japonicus (Bloch, 1791) & $\begin{array}{l}\text { Japanese } \\
\text { threadfin bream }\end{array}$ & $\begin{array}{l}\text { A, B, } \\
\text { C, D }\end{array}$ & $\mathrm{LC}$ & $\mathrm{H}$ & $\mathrm{C}$ \\
\hline 101 & & N. bipunctatus (Valenciennes, 1830) & $\begin{array}{l}\text { Delagoa } \\
\text { threadfin bream }\end{array}$ & A, B & $\mathrm{LC}$ & $\mathrm{H}$ & $\mathrm{C}$ \\
\hline 102 & Platycephalidae & Platycephalus indicus (Linnaeus, 1758) & Bartail flathead & A, B & DD & $\mathrm{H}$ & $\mathrm{C}, \mathrm{Gf}$ \\
\hline 103 & Polynemidae & Polynemus paradiseus Linnaeus, 1758 & $\begin{array}{l}\text { Paradise } \\
\text { threadfin }\end{array}$ & A, B & $\mathrm{LC}$ & $\mathrm{H}$ & $\mathrm{C}$ \\
\hline 104 & & $\begin{array}{l}\text { Polydactylus plebei- } \\
\text { us (Broussonet, 1782) }\end{array}$ & $\begin{array}{l}\text { Striped } \\
\text { threadfin }\end{array}$ & A, B & NE & $\mathrm{H}$ & $\mathrm{C}, \mathrm{Gf}$ \\
\hline 105 & & $\begin{array}{l}\text { Eleutheronema tetradac- } \\
\text { tylum (Shaw, 1804) }\end{array}$ & $\begin{array}{l}\text { Fourfinger } \\
\text { threadfin }\end{array}$ & $\begin{array}{l}\text { A, B, } \\
\text { C, D }\end{array}$ & NE & $\mathrm{H}$ & $\mathrm{C}, \mathrm{Gf}$ \\
\hline 106 & & $\begin{array}{l}\text { Leptomelanosoma indic- } \\
\text { um (Shaw, 1804) }\end{array}$ & Indian threadfin & $\mathrm{A}, \mathrm{B}$ & NE & $\mathrm{H}$ & $\mathrm{C}, \mathrm{Gf}$ \\
\hline 107 & Priacanthidae & Priacanthus hamrur (Forsskål, 1775) & $\begin{array}{l}\text { Moontail } \\
\text { bullseye }\end{array}$ & $\begin{array}{l}\text { A, B, } \\
\text { C, D }\end{array}$ & $\mathrm{LC}$ & $\mathrm{H}$ & $\mathrm{C}, \mathrm{Aq}$ \\
\hline 108 & Psettodidae & $\begin{array}{l}\text { Pseudorhombus arsi- } \\
\text { us (Hamilton, 1822) }\end{array}$ & $\begin{array}{l}\text { Largetooth } \\
\text { flounder }\end{array}$ & A, B & $\mathrm{NE}$ & $\mathrm{H}$ & $\mathrm{C}, \mathrm{Gf}$ \\
\hline
\end{tabular}


109

\section{0}

11

111

112

113

114

115

116

117

118

119

120

121

122

123

124

125

126

127

128

128

129

130

131

132

133

134

135

136

137
Psettodes erumei (Bloch

\& Schneider, 1801)

Rachycentridae Rachycentron canad-

um (Linnaeus, 1766)

Scatophagidae Scatophagus argus (Linnaeus, 1766)

Sciaenidae

Atrobucca nibe (Jordan \&

Thompson, 1911)

Bababa chaptis (Hamilton, 1822)

Chrysochir aureus (Richardson, 1846)

Daysciaena albida (Cuvier, 1830)

Dendrophysa russelii (Cuvier, 1829)

Johnius belangerii (Cuvier, 1830)

Johnius carutta Bloch, 1793

J. dussumieri (Cuvier, 1830)

Macrospinosa cuja (Hamilton, 1822)

Nibea coibor (Hamilton, 1822)

N. maculate (Bloch \& Sch-

neider, 1801)

Otolithes ruber (Bloch \&

Schneider, 1801)

Protonibea diacanthus (Lacepède, 1802)

Scombridae

Auxis thazard (La

Auxis thazard (Lacepède, 1800)

Euthynnus affinis (Cantor, 1849)

Katsuwonus pelamis (Linnaeus, 1758) Skipjack tuna

Rastrelliger kanagurta (Cuvier, 1816)

Scomberomorus commer-

son (Lacepède, 1800)

S. guttatus (Bloch \& Schneider, 1801) IndoPacific

king mackerel

Streaked seerfish A, B

$\mathrm{C}, \mathrm{D}$

A, B

C, D

A, B, LC
C, D

A, B,

C, D

C, D

$\mathrm{A}, \mathrm{B}, \quad \mathrm{LC} \quad \mathrm{H} \quad \mathrm{C}, \mathrm{Ac}$

$\mathrm{C}, \mathrm{D}$

A, B,

A, B NE H C

A, B DD $\mathrm{H} \quad \mathrm{C}$

$A, B, \quad N E \quad H \quad C$

C, D

A, B, NE H C

C, D

$\mathrm{A}, \mathrm{B}, \quad \mathrm{NE} \quad \mathrm{H} \quad \mathrm{C}$

C, D

A, B, NE H C

C, D

A, B NE H C

A, B NE H C

B, C NE H $\mathrm{C}$

$A, B, \quad N E \quad H \quad$ Ss

$\mathrm{C}, \mathrm{D}$

C, D

A, B NE $\quad \mathrm{H} \quad \mathrm{C}, \mathrm{Gf}$

A, B NE $\mathrm{H} \quad \mathrm{C}$

A, B LC $\quad \mathrm{H} \quad$ C, Gf

A, B, LC Pn C, Gf

C, D

A, B, NT Pn $\quad$, Gf

C, D

$\mathrm{A}, \mathrm{B}, \quad \mathrm{DD} \quad \mathrm{H} \quad \mathrm{C}, \mathrm{Gf}$

$\mathrm{C}, \mathrm{D}$

S. lineolatus (Cuvier, 1829)

Thunnus albacares (Bonnaterre, 1788) Yellowfin tuna

Bigeye tuna

A, B VU H

C, Gf

T. tonggol (Bleeker, 1851)

Serranidae

Cephalopholis aurantia $(\mathrm{Va}-$

Longtail tuna

A, B DD H

C, Gf lenciennes, 1828)

Cephalopholis formosa (Shaw, 1812)

Cromileptes altivelis ( $\mathrm{Va}$ -

lenciennes, 1828)
Golden hind A

LC H C

Blue lined hind A, B, LC $\quad \mathrm{H} \quad$ Sf

C, D

Humpback A DD H $\quad$ C, Ac grouper 


\begin{tabular}{|c|c|c|c|c|c|c|c|}
\hline 138 & & Epinephelus areolatus (Forsskål, 1775) & Areolate grouper & B & $\mathrm{LC}$ & $\mathrm{H}$ & $\mathrm{C}$ \\
\hline 139 & & E. bleekeri (Vaillant, 1878) & $\begin{array}{l}\text { Dusky tail } \\
\text { grouper }\end{array}$ & B & $\mathrm{DD}$ & $\mathrm{H}$ & $\mathrm{C}, \mathrm{Ac}$ \\
\hline 140 & & E. coioides (Hamilton, 1822) & $\begin{array}{l}\text { Orange-spot- } \\
\text { ted grouper }\end{array}$ & $\begin{array}{l}\text { A, B, } \\
\text { C, D }\end{array}$ & $\mathrm{LC}$ & $\mathrm{H}$ & $\mathrm{C}, \mathrm{Ac}$ \\
\hline 141 & & $\begin{array}{l}\text { E. latifasciatus (Temminck } \\
\text { \& Schlegel, 1842) }\end{array}$ & Striped grouper & $\mathrm{B}$ & $\mathrm{LC}$ & $\mathrm{H}$ & $\mathrm{C}$ \\
\hline 142 & & E. radiatus (Day, 1868$)$ & $\begin{array}{l}\text { Oblique-band- } \\
\text { ed grouper }\end{array}$ & $\mathrm{B}$ & $\mathrm{LC}$ & $\mathrm{H}$ & $\mathrm{C}$ \\
\hline 143 & Siganidae & Siganus stellatus (Forsskål, 1775) & $\begin{array}{l}\text { Brown-spot- } \\
\text { ted spinefoot }\end{array}$ & $\mathrm{A}, \mathrm{B}$ & $\mathrm{LC}$ & Vn & $\mathrm{C}$ \\
\hline 144 & Sillaginidae & Sillago sihama (Forsskål, 1775) & Silver sillago & $\begin{array}{l}\text { A, B, } \\
\text { C, D }\end{array}$ & $\mathrm{LC}$ & $\mathrm{H}$ & $\mathrm{C}$ \\
\hline 145 & Soleidae & Synaptura albomaculata Kaup, 1858 & Kaup's sole & $A, B$ & $\mathrm{NE}$ & $\mathrm{H}$ & $\mathrm{C}$ \\
\hline 146 & & S. commersonnii (Lacepède, 1802) & $\begin{array}{l}\text { Commer- } \\
\text { son's sole }\end{array}$ & $\begin{array}{l}\text { A, B, } \\
\text { C, D }\end{array}$ & $\mathrm{NE}$ & $\mathrm{H}$ & $\mathrm{C}$ \\
\hline 147 & Sphyraenidae & Sphyraena obtusata Cuvier, 1829 & Barracuda & $\begin{array}{l}\text { A, B, } \\
\text { C, D }\end{array}$ & $\mathrm{NE}$ & $\mathrm{H}$ & $\mathrm{C}, \mathrm{Gf}$ \\
\hline 148 & Stromateidae & Pampus argenteus (Euphrasen, 1788) & Silver pomfret & $\begin{array}{l}\text { A, B, } \\
\text { C, D }\end{array}$ & $\mathrm{NE}$ & $\mathrm{H}$ & $\mathrm{C}$ \\
\hline 149 & & P. chinensis (Euphrasen, 1788) & $\begin{array}{l}\text { Chinese silver } \\
\text { pomfret }\end{array}$ & $\begin{array}{l}\mathrm{A}, \mathrm{B} \\
\mathrm{C}, \mathrm{D}\end{array}$ & $\mathrm{NE}$ & $\mathrm{H}$ & $\mathrm{C}$ \\
\hline 150 & Terapontidae & Terapon jarbua (Forsskål, 1775) & Jarbua terapon & $\begin{array}{l}\text { A, B, } \\
\text { C, D }\end{array}$ & $\mathrm{LC}$ & $\mathrm{H}$ & $\mathrm{C}$ \\
\hline 151 & & T. puta Cuvier, 1829 & $\begin{array}{l}\text { Small-scaled } \\
\text { terapon }\end{array}$ & $A, B$ & $\mathrm{NE}$ & $\mathrm{H}$ & $\mathrm{C}$ \\
\hline 152 & & T. theraps Cuvier, 1829 & $\begin{array}{l}\text { Large-scaled } \\
\text { terapon }\end{array}$ & $A, B$ & $\mathrm{LC}$ & $\mathrm{H}$ & $\mathrm{C}$ \\
\hline 153 & Trichiuridae & $\begin{array}{l}\text { Eupleurogrammus glosso- } \\
\text { don (Bleeker, 1860) }\end{array}$ & $\begin{array}{l}\text { Long tooth } \\
\text { hairtail }\end{array}$ & $\begin{array}{l}\mathrm{A}, \mathrm{B} \\
\mathrm{C}, \mathrm{D}\end{array}$ & $\mathrm{NE}$ & $\mathrm{H}$ & $\mathrm{C}$ \\
\hline 154 & & E. muticus (Gray, 1831) & $\begin{array}{l}\text { Small head } \\
\text { hairtail }\end{array}$ & $\begin{array}{l}\text { A, B } \\
\text { C, D }\end{array}$ & $\mathrm{NE}$ & $\mathrm{H}$ & $\mathrm{C}$ \\
\hline 155 & & Lepturacanthus savala (Cuvier, 1829) & Savalai hairtail & $\begin{array}{l}\text { A, B, } \\
\text { C, D }\end{array}$ & $\mathrm{NE}$ & $\mathrm{H}$ & $\mathrm{C}$ \\
\hline 156 & & L. pantului (Gupta, 1966) & $\begin{array}{l}\text { Coroman- } \\
\text { del hairtail }\end{array}$ & $A, B$ & $\mathrm{DD}$ & $\mathrm{H}$ & $\mathrm{C}$ \\
\hline 157 & & Trichiurus gangeticus Gupta, 1966 & Ganges hairtail & $\begin{array}{l}\text { A, B, } \\
\text { C, D }\end{array}$ & $\mathrm{NE}$ & $\mathrm{H}$ & $\mathrm{C}$ \\
\hline 158 Siluriformes & Ariidae & Arius arius (Hamilton, 1822) & $\begin{array}{l}\text { Threadfin } \\
\text { sea catfish }\end{array}$ & $\begin{array}{l}\text { A, B, } \\
\text { C, D }\end{array}$ & $\mathrm{NE}$ & $\operatorname{Tr}$ & $\mathrm{C}$ \\
\hline 159 & & A. jella Day, 1877 & $\begin{array}{l}\text { Blackfin sea } \\
\text { catfish }\end{array}$ & $\begin{array}{l}\text { A, B, } \\
\text { C, D }\end{array}$ & $\mathrm{NE}$ & $\operatorname{Tr}$ & $\mathrm{C}$ \\
\hline 160 & & A. maculatus (Thunberg, 1792) & Spotted catfish & $\begin{array}{l}\text { A, B, } \\
\text { C, D }\end{array}$ & $\mathrm{NE}$ & $\operatorname{Tr}$ & $\mathrm{C}$ \\
\hline 161 & & $\begin{array}{l}\text { Nemapteryx caelata (Va- } \\
\text { lenciennes, 1840) }\end{array}$ & Engraved catfish & $A, B$ & $\mathrm{NE}$ & $\operatorname{Tr}$ & $\mathrm{C}$ \\
\hline 162 & & $\begin{array}{l}\text { Plicofollis dussumieri (Va- } \\
\text { lenciennes, 1840) }\end{array}$ & $\begin{array}{l}\text { Blacktip sea } \\
\text { catfish }\end{array}$ & B & $\mathrm{LC}$ & $\operatorname{Tr}$ & Ss \\
\hline 163 & & Sciades sona (Hamilton, 1822) & Sona sea catfish & B & LC & $\operatorname{Tr}$ & C \\
\hline 164 & Plotosidae & Plotosus canius Hamilton, 1822 & Gray eel-catfish & $\mathrm{B}$ & $\mathrm{NE}$ & $\mathrm{Vn}$ & $\mathrm{C}$ \\
\hline
\end{tabular}




\begin{tabular}{llllllll}
\hline 165 Tetraodontiformes & Diodontidae & Diodon holocanthus Linnaeus, 1758 & $\begin{array}{l}\text { Long spined } \\
\text { porcupinefish }\end{array}$ & A, B & LC & Pn & C, Aq \\
166 & Tetraodontidae & Arothron hispidus (Linnaeus, 1758) & $\begin{array}{l}\text { White-spot- } \\
\text { ted puffer }\end{array}$ & A, B & LC & Pn & C, Aq \\
167 & Chelonodon patoca (Hamilton, 1822) & $\begin{array}{l}\text { Milkspot- } \\
\text { ted puffer }\end{array}$ & $\begin{array}{l}\text { A, B, } \\
\text { C, D }\end{array}$ & LC & Pn & C \\
168 & & $\begin{array}{l}\text { Lagocephalus guentheri } \\
\text { Miamond- } \\
\text { back puffer }\end{array}$ & $\begin{array}{l}\text { A, B, } \\
\text { MirandaRibeiro, } 1915\end{array}$ & LC & H & Ss \\
170 & Takifugu oblongus (Bloch, 1786) & Lattice blaasop & A, B, & LC & H & Ss \\
171 & Triacanthidae & Triacanthus biaculeatus (Bloch, 1786) & $\begin{array}{l}\text { Short-nosed } \\
\text { tripodfish }\end{array}$ & A, B & NE & H & C \\
& Ostraciidae & Tetrosomus gibbosus (Linnaeus, 1758) & $\begin{array}{l}\text { Humpback } \\
\text { turretfish }\end{array}$ & B & LC & Vn & C, Aq
\end{tabular}

Station: A = Machilipatnam; B = Nizampatnam; C = Vodarevu; D = Pakala. IUCN Status: VU = Vulnerable; LC = Least Concern; $\mathrm{NE}=$ Not Evaluated; DD = Data Deficient; NT = Near Threatened; EN=Endangered. Threat to Humans: Harmless $=\mathrm{H}$; Traumatogenic $=$ Tr; Venomous $=$ Vn; Poisonous $=$ Pn. Human Use: $\mathrm{C}=$ Commercial; F $=$ Fisheries; Aq = Aquarium, Ss $=$ Scientific study; $\mathrm{Sf}=$ Subsistence fisheries; $\mathrm{Ac}=$ Aquaculture; $\mathrm{Gf}=\mathrm{Gamefish} ; \mathrm{Ukn}=$ Unknown.

\section{DivERSITY INDICES}

The species diversity indices viz., i) Shannon-Wiener species diversity index (H') (Shannon and Wiener, 1949), ii) Margalef richness index (SR) (Margalef, 1968) and iii) Simpson's dominance index (D) (Simpson, 1949) were analyzed using the PAST (Palaeontological Statistics) software (Version 2.02).

\section{Shannon-Weiner's diversity index;}

$$
\mathrm{H}^{\prime}=\sum_{\mathrm{i}} 1 \mathrm{P}_{\mathrm{i}} \log _{\mathrm{e}} \mathrm{P}_{\mathrm{i}}
$$

where, $\mathrm{H}^{\prime}=$ species diversity in bits of information per individual; $\mathrm{P}_{\mathrm{i}}=\mathrm{ni} / N$ (proportion of the samples belonging to the species; $\mathrm{ni}=$ number of individuals in all the samples; $\mathrm{N}=$ total number of individuals in the collection).

\section{Margalef Species richness index;}

$$
\mathrm{SR}=(\mathrm{S}-1) / \log \mathrm{N}
$$

where, $\mathrm{S}=$ total number of species, and $\mathrm{N}=$ total number of individuals in the collection.

\section{Simpson's Dominance index;}

$$
\mathrm{D}=\sum_{\mathrm{i}=1}^{\mathrm{S}}\left(\frac{\mathrm{ni}}{\mathrm{N}}\right)^{2}
$$

where, $\mathrm{ni}=$ number of individuals in the 'each' species, $\mathrm{N}$ $=$ total number of individuals, $\mathrm{S}=$ total number of species.

\section{RESULTS AND DISCUSSION}

A comprehensive checklist of the species of fish recorded, the common names, site of occurrence at landing sites, IUCN status, risk to humans and usage are presented in Table 2. In the present study, a total of 171 species belonging to 14 orders, 63 families and 128 genera were recorded from four fish landing stations of Andhra Pradesh viz. Nizampatnam, Machilipatnam, Vodarevu, and Pakala. Of the total species, 17 species belonged to Chondrichthyes/ Elasmobranchii, and 154 species belonged to Osteichthyes/ Actinopterygii. The total number of species in various genera, families and orders recorded are presented in Table 3.

In India, mention may be made to the earlier works of $\mathrm{Su}-$ darsan (1988) which provided key to 273 species of fish in trawl catches off Visakhapatnam. Krishnan and Mishra (1993) reported 114 species from Kakinada, east coast of India. Sujatha (1995) reported 228 fish species belonging to 68 families from Visakhapatnam, Andhra Pradesh, southeast coast of India. Barman et al. (2004) documented 580 fish species under 292 genera, 121 families, and 27 orders from Andhra Pradesh. Gibinkumar et al. (2012) reported 191 fish species belonged to 12orders, 59 families and 109 genera from Cochin, southwest coast of India. Sambandamoorthy et al. (2015) reported123 fish species belonging to 13 orders, 49 families and 2 genera from the southeast coast of India.

The variation in the species number reported by different workers over a period of time could be due to the change in climate or the prevailing environmental conditions. In India, Andhra Pradesh is the second most cyclone-affected state (Babu et al., 2014; Bharti et al., 2017). During the study period, the impact of many cyclonic storms such as Titli, Gaja, and Phethai experienced on this coast might 
have prevented the fish from venturing into the sea resulting in less number of species recorded. The considerable decline might also be due to the reduction in the days of fishing due to cyclonic storms in Andhra Pradesh (FRAD, 2019).

Table 3: Number of Orders, Families, Genera and Species recorded in the present study.

\begin{tabular}{|lllll} 
S. No & Order & Family & Genus & Species \\
\hline 1 & Carcharhiniformes & 2 & 5 & 7 \\
\hline 2 & Myliobatiformes & 2 & 4 & 5 \\
\hline 3 & Orectolobiformes & 1 & 1 & 1 \\
\hline 4 & Rhinopristiformes & 2 & 2 & 2 \\
\hline 5 & Torpediniformes & 2 & 2 & 2 \\
\hline 6 & Anguilliformes & 3 & 4 & 4 \\
\hline 7 & Aulopiformes & 1 & 2 & 2 \\
\hline 8 & Beloniformes & 3 & 4 & 4 \\
\hline 9 & Clupeiformes & 4 & 13 & 22 \\
\hline 10 & Elopiformes & 1 & 1 & 1 \\
\hline 11 & Mugiliformes & 1 & 3 & 4 \\
\hline 12 & Perciformes & 35 & 75 & 103 \\
\hline 13 & Siluriformes & 2 & 5 & 7 \\
\hline 14 & Tetraodontiformes & 4 & 7 & 7 \\
\hline & Total & 63 & 128 & 171 \\
\hline
\end{tabular}

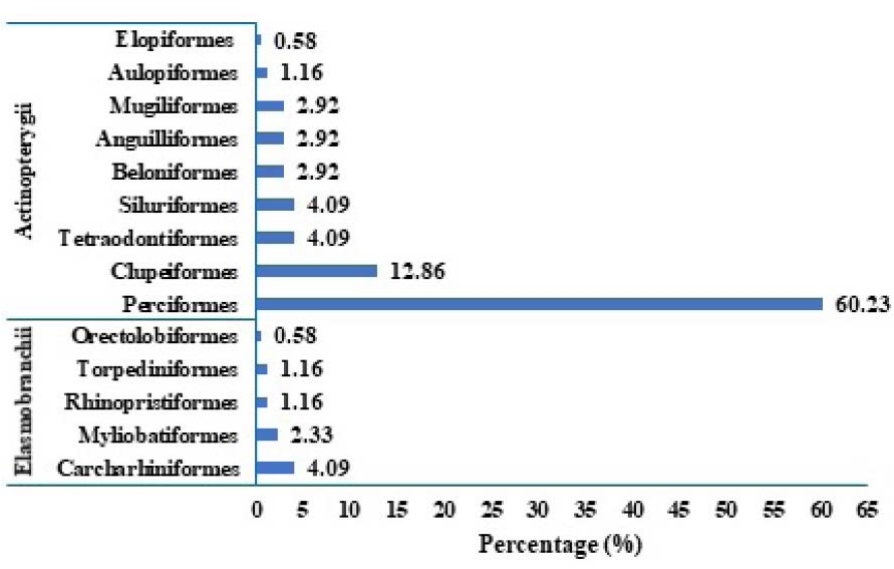

Figure 3: Percentage abundance of various Orders of fish documented at four landing stations.

The relative percentage abundance of various orders of fish and the respective families, genera and species recorded from landing stations are depicted in Figures 3 and 4. The number of species recorded from various orders of fish is: Perciformes (60.23\%) with 103 species, followed by Clupeiformes (12.86\%) with 22 species, Siluriformes, Carcharhiniformes and Tetraodontiformes (4.09\%) with 7 species each, Myliobatiformes (2.92\%) with 5 species, Anguilliformes, Beloniformes, and Mugiliformes (2.33\%) each with 4 species, Aulopiformes, Rhinopristiformes and Torpediniformes (1.16\%) with 2 species each, and Elopi- formes and Orectolobiformes (0.58\%) with 1 species each. Among the orders, Perciformes is dominant (60.23\%) representing 35 families with 103 species, a trend that is similar across various independent studies. Kar et al. (2017) reported 157 perciform species from coastal waters of West Bengal, and Jayaprabha et al. (2018) recorded 113 perciform species from Tamil Nadu, southeast coast of India.

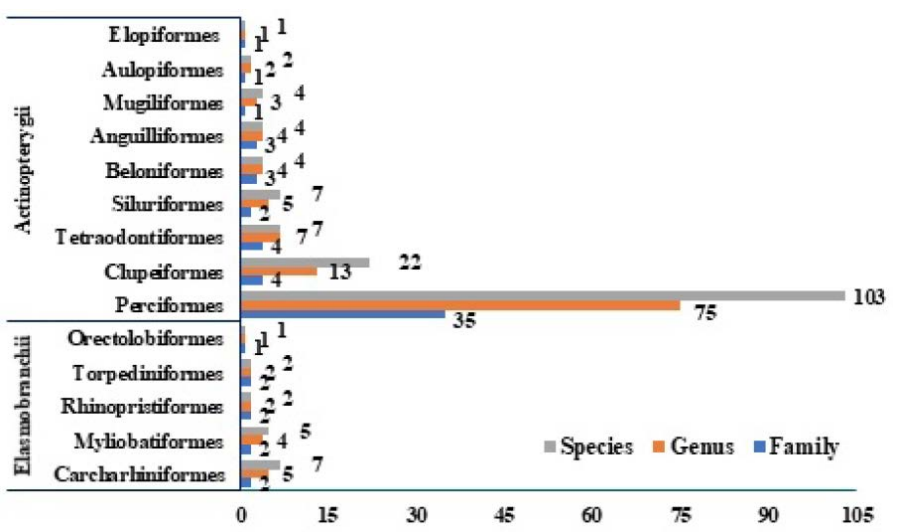

Figure 4: Relative Abundance of Species, Genera, Families and Orders of Fish recorded at four landing stations.

Table 4: Fish taxa in the four landing stations along coastal Andhra Pradesh.

\begin{tabular}{lllll} 
Taxa & \multicolumn{4}{l}{ Landing Stations } \\
& $\mathrm{A}$ & $\mathrm{B}$ & $\mathrm{C}$ & $\mathrm{D}$ \\
Class & 2 & 2 & 2 & 2 \\
Order & 12 & 13 & 9 & 7 \\
Family & 51 & 59 & 31 & 30 \\
Genus & 106 & 118 & 69 & 67 \\
Species & 143 & 159 & 91 & 86
\end{tabular}

$\mathrm{A}=$ Machilipatnam; $\mathrm{B}=$ Nizampatnam; $\mathrm{C}=$ Vodarevu; $\mathrm{D}=$ Pakala

Considering the station-wise landing data, the number of species recorded at station B (159) and A (143) are relatively higher than those at stations $\mathrm{C}(91)$ and $\mathrm{D}(86)(\mathrm{Ta}-$ ble 4). The majority of species numbering 73 (42\%) were recorded in all four stations and in particular, 60 species (35\%) were common in both $\mathrm{A}$ and $\mathrm{B}$ stations. This variation in species number may be due to the availability of mechanized trawl catches and fishing harbour facilities at stations $\mathrm{A}$ and $\mathrm{B}$ rather than at $\mathrm{C}$ and $\mathrm{D}$. It is evident that among 171 species, commercial fishes are 153, and the remaining fishes are used for aquarium, aquaculture, fisheries, and sports and for research studies (Table 2).

The Indian coastline contributes rich diverse fishery resources having substantial economic value (Darwin and Padmavathi, 2020). Despite commercial significance, no attempt has been made so far to know the conservation status for these fishes. According to IUCN red list of conservation status, among 171 fish species, 78 species (45.61\%) 
are marked as Least Concern (LC), 65 species (38\%) as Not Evaluated, 13 species (7.60\%) as Data Deficient (DD) due to lack of adequate information, 6 species (3.5\%) each as Near Threatened (NT) and Vulnerable (VU) and 3 species $(1.75 \%)$ as Endangered (EN) (Figure 5). Out of 171 species, 65 species are not yet evaluated indicating an urgent need of conservation studies in these areas for establishing sustainable marine fisheries along the coast of Bay of Bengal. The conservation of fish also requires further studies on their complex life cycles.

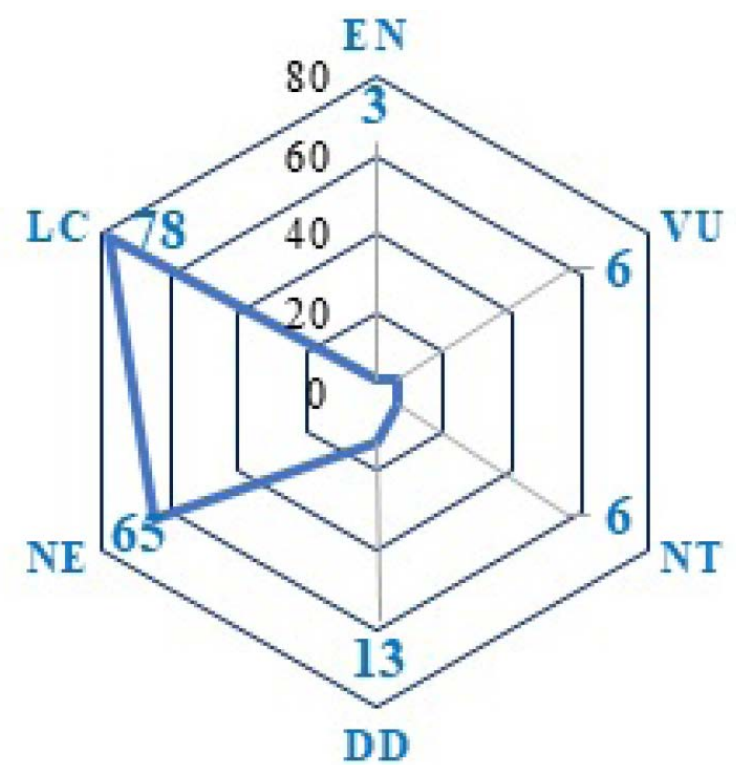

Figure 5: IUCN status of fishes reported in landing stations.

Overfishing is one of the severe concerns affecting the community structure of fish with threatening and extinction of species (Jackson et al., 2001). Since the White cheek shark, Carcharbinus dussumieri, Broadfin Shark, Lamiopsis temminckii and Longheaded eagle ray, Aetobatus flagellum are recognized as Endangered (EN) species, immediate steps should be taken to stop catching these fish species for at least few years to rise their number to a reasonable level. Similarly, Blacktip shark Carcharbinus limbatus, Hammer head shark Sphyrna zygaena, Honeycomb stingray Himantura uarnak, Sharp nose stingray Maculabatis gerrardi, Giant guitarfish Rhynchobatus djiddensis, and Bigeye tuna Thunnus obesus are identified as Vulnerable (VU) fish species. Therefore, effective steps are essential to conserve the species and maintain harmony in the marine community and ensure sustainable management practice in the near future.

Different diversity indices were calculated with respect to four different sampling stations of coastal Andhra Pradesh. The values of Shannon-Wiener species diversity index $\left(\mathrm{H}^{\prime}\right)$, and Margalef species richness index (SR) and Simpson dominance index value (D) were presented in Table 5. The maximum Shannon Diversity Index (5.069) was found at Nizampatnam station and the minimum (4.454) at Pakala.
The highest Margalef species richness index (SR) of 31.17 was observed at Nizampatnam station whereas the lowest value of 19.08 at Pakala station. The maximum Simpson dominance value (D) of 0.994 was noticed at Nizampatnam and the minimum 0.988 in Pakala landing site.

Table 5: Fish Diversity Indices in Four Sampling Stations along Coastal Andhra Pradesh.

\begin{tabular}{llll} 
Sampling Stations & \multicolumn{3}{l}{ Diversity indices } \\
& H' & SR & D \\
Machilipatnam & 4.963 & 28.61 & 0.993 \\
\hline Nizampatnam & 5.069 & 31.17 & 0.994 \\
Vodarevu & 4.511 & 19.95 & 0.989 \\
\hline Pakala & 4.454 & 19.08 & 0.988 \\
\hline H' $=$ diversity index; SR = species richness; D = dominance
\end{tabular}

Two main components involved in diversity of species are the species richness and distribution of individuals among species where the evaluation of species richness is complex (Williamson, 1973). In Shannon-Weiner index, the water and soil in aquatic environment has been considered as very poor quality when it is $<1$, poor quality $1-2$, moderate quality $2-3$, good quality $3-4$. In the present study, the Shannon index was within the range of $5.069-4.454$ which indicated that these study stations are in favorable conditions. A community becomes more divergent as the stress increases and consequently the species diversity decreases with unfavourable environmental conditions. A community with relatively few species represents that the environment is under stress (Plafkin et al., 1989). Species richness (SR) and Dominance (D) indices were found to be highest in Nizampatnam followed by Machilipatnam, Vodarevu and Pakala. The indices values were highest in Nizampatnam and Machilipatnam stations which indicate favourable conditions for fish abundance. When the temporal variation was compared, the species dominance among all the stations did not vary for a greater magnitude. The reason for more number of species at Nizampatnam and Machilipatnam stations might be due to the influx of estuarine species through Krishna river water to the marine fish stocks in between these landing stations. In addition to this, the ecological conditions have an effect on the distribution of fish species.

\section{CONCLUSION}

The present study provides information on marine fish diversity along the four landing stations of coastal Andhra Pradesh. It is evident that the recorded 171 species of fish upholds a vital fishery along the southeast coast of India. However, the species reported in the present study are lesser than those reported by Sudarsan (1981), Sujatha (1995), Barman et al. (2004), Gibinkumar et al. (2012), Kar et al. (2017). Most of the threats to the fish diversity in India are 
due to several natural and anthropogenic stress factors (Das et al., 2004; Gopi and Mishra, 2015; Joshi et al., 2015). The non-availability and less availability of some species indicate an alarming decline of marine fish diversity in the surveyed area and perhaps in the country as a whole. It is imperative to undertake a state-wide analysis of qualitative and quantitative availability of natural fish resources and the conservation status of marine fish. Therefore, a detailed long-term investigation of marine fish is needed to protect and conserve the species through effective policy decisions. This will pave the way for better conservation of natural fish diversity and benefit the mankind for more sustainable livelihood approach in the near future.

\section{ACKNOWLEDGMENTS}

The authors are thankful to the University Grants Commission (UGC), New Delhi, Government of India, for granting BSR (Basic Scientific Research) fellowship to Mr. Darwin Chatla and the authorities of Acharya Nagarjuna University for providing the necessary amenities to complete this work in the Department of Zoology \& Aquaculture.

\section{AUTHOR CONTRIBUTIONS}

Both the authors contributed equally.

\section{CONFLict Of InTEREST}

The authors have no conflicts of interest to the content of this article.

\section{REFERENCES}

- Babu KR, Ranjan TJ, Reddy KS, Raju MR (2014). Impact of the tropical cyclonic storm 'Hudhud' on northeast coastal waters of Visakhapatnam. Am. J. Mar. Sci. 2(3):63-6.

- Barman RP, Kar S, Mukherjee P (2004). Marine and estuarine fishes. In: Director (ed). State Fauna Series 5. Fauna of Andhra Pradesh, Part-2, (Zool. Surv. India, Kolkata).101-54

- Bharti V, Mini KG, Sathianandan TV, Pugazhendi D, Manjeesh R, Augustine SK (2017). Marine fish landings in Andhra Pradesh during 2016 - An overview. Mar. Fish. Infor. Sew. T \& E Ser., No. 233:19-20

- Chatla D, Padmavathi P, Srinu G (2020). Wastewater Treatment Techniques for Sustainable Aquaculture. In: S. Ghosh (eds) Waste Management as Economic Industry Towards Circular Economy. Springer, Singapore. https://doi.org/10.1007/978981-15-1620-7_17

- CMFRI (2012). Marine Fisheries Census 2010 Part II. 3 Andhra Pradesh. (Central Marine Fisheries Research Institute, Kochi) http://eprints.cmfri.org.in/id/eprint/8999.

-DADF (2019). Department of Animal Husbandry Dairying and Fisheries, Annual Report, 2018-19. https://dahd.nic.in/ documents/reports.

-Darwin C, Padmavathi P (2020). Diversity and current status of grouper fish, Epinephelus Bloch, 1793 in Indian coastal waters.
Adv. Anim. Vet. Sci. 8(11):1161-9. https://doi.org/10.17582/ journal.aavs/2020/8.11.1161.1169

-Das P, De SP, Bhowmick RM, Nandy AC, Pandit PK, Sengupta RC, Thakurta SC (2004). Diminishing trend of fish species diversity in West Bengal: field study. Fish. Chimes 24 (1):738

-FRAD (2019). Marine Fish Landings in India 2018. Technical Report. ICAR-Central Marine Fisheries Research Institute, Kochi. http://eprints.cmfri.org.in/id/eprint/14753.

-FRAD, CMFRI (2020). Marine Fish Landings in India 2019. Technical Report. ICAR-Central Marine Fisheries Research Institute, Kochi.

- Fricke R, Eschmeyer WN, Van der Laan R (2020). Eschmeyer's Catalog of Fishes: Genera, Species, References. World Wide Web electronic publication www.calacademy.org.

- Froese R, Pauly D (2020). FishBase. World Wide Web electronic publication. www.fishbase.org.

- Gibinkumar TR, Sabu S, Pravin P, Boopendranath MR (2012). Bycatch characterization of shrimp trawl landings off southwest coast of India. Fish. Technol. 49: 132-140.

- Gopi KC, Mishra SS (2015). Diversity of marine fish of India. In: K. Venkataraman, C. Sivaperuman (edS) Marine Faunal Diversity in India. Taxonomy, Ecology and Conservation, (Academic Press, Elsevier Inc., USA), 171-193. https://doi. org/10.1016/B978-0-12-801948-1.00012-4

-IUCN (2020). International Union for Conservation of Nature. World Wide Web electronic publication. www.iucn.org.

- Jackson JB, Kirby MX, Berger WH, Bjorndal K, Botsford LW, Bourque BJ (2001). Historical overfishing and the recent collapse of coastal ecosystems. Sci. 293 (5530): 629-37. https://doi.org/10.1126/science.1059199

- Jayaprabha N,Purusothaman S, Srinivasan M(2018). Biodiversity of coral reef associated fishes along southeast coast of India. Reg. Stud. Mar. Sci. 18: 97-105. https://doi.org/10.1016/j. rsma.2017.12.010

-Joshi KK, Varsha MS, Sruthy VL (2015). Marine biodiversity of India-status and challenges. In: K.K. Joshi, M.S. Varsha, V.L. Sruthy et al (eds) Summer School on Recent Advances in Marine Biodiversity Conservation and Management, Manual, (Central Marine Fisheries Research Institute, Kochi), 9-12. http://eprints.cmfri.org.in/id/eprint/12067

- Kar A, Raut SK, Bhattacharya M, Patra S, Das BK, Patra BC (2017). Marine fishes of West Bengal coast, India: Diversity and conservation preclusion. Reg. Stud. Mar. Sci.16:56-66. https://doi.org/10.1016/j.rsma.2017.08.009

-Krishnan S, Mishra SS (1993). On a collection of fish from Kakinada-Gopalpur sector of the east coast of India. Rec. Zool. Surv. India. 93(1-2): 201-240

-Margalef R (1968). Perspectives in Ecological Theory. Chicago II. University of Chicago Press, Chicago. 111.

- Nelson JS, Grande TC, Wilson MV (2016). Fishes of the World. John Wiley \& Sons, Inc. Hoboken. https://doi. org/10.1002/9781119174844

- Plafkin JL (1989). Rapid bioassessment protocols for use in streams and rivers: benthic macroinvertebrates and fish. United States Environmental Protection Agency, Office of Water.

-Pyle RL, Kosaki RK, Pinheiro HT, Rocha LA, Whitton RK, Copus JM (2019). Fishes: biodiversity. In: Loya Y, Puglise K, Bridge T (ed) Mesophotic Coral Ecosystems. Coral Reefs of the World. 12: 749-777. https://doi.org/10.1007/978-3319-92735-0_40

- Rajan PT (2018). Marine fishery resources and species diversity 
of tropical waters. In: Sivaperuman, C, Velmurugan A, Singh AK Jaisankar I (ed) Biodiversity and Climate Change Adaptation in Tropical Islands. (Academic Press), 323-354. https://doi.org/10.1016/B978-0-12-813064-3.00011-9

- Rao GS, Rohit P, Maheswarudu G, Rajkumar U (2008). Marine fisheries of Andhra Pradesh-an appraisal. Mar Fish Infor Sew T \& E Ser 196:1-5.

-Sambandamoorthy P, Nagamuthu J, Arumugam S, Perumal M (2015). Diversity and trophic level of ichthyofauna associated with the trawl bycatches of Cuddalore and Parangipettai, south-east coast of India. Mar. Biodivers. Rec.8: 1-8. https:// doi.org/10.1017/S1755267215000159

-Shannon CE, Weaver's W (1949). The mathematical theory of communications. Urbana, University of Illinois. 117

- Simpson EH (1949). Measurement of diversity. Nature. 163:68. https://doi.org/10.1038/163068a0

- Sreedhar U, Prakash RR, Rajeswari G (2010) Present scenario of the coastal and deep water. In: Meenakumari B, Boopendranath MR, Edwin L, Sankar TV, Gopal N, Ninan G (ed) Coastal Fishery Resources of India: Conservation and Sustainable Utilisation. (Society of Fisheries Technologists (India), Cochin). 77-89.
- Sudarsan D, Somvanshi VS (1988). Fishery resources of Indian $\mathrm{EEZ}$ with special reference to upper east coast. Bulletin of fishery survey of India. 16: 1-27.

- Sujatha K (1995). Finfish constituents of trawl by-catch off Visakhapatnam. Fish. Technol. 32: 56-56.

- Talwar PK, Kacker RK (1984). Commercial sea fishes of India. Zool. Surv. India. 1-997

-Varadharajan D, Soundarapandian P (2015). Fishery Potential of Mallipattinam Coast, South East Coast of India. J. Earth. Sci. Clim. Change. 6 (6):1-4. https://doi.org/10.4172/21577617.1000285

-WAPCOS (2017). Rapid Need Assessment \& Pre-Feasibility Study Report for Development of Fishery Harbours at Nizampatnam in Andhra Pradesh. Government of Andhra Pradesh, Department of Fisheries. Wapcos 1td. Gurgaon, pp $1-42$.

-Williamson M (1973). Species diversity in ecological communities. The mathematical theory of the dynamics of biological populations. Academic, London, 325-336.

-WoRMS (2020). World Register of Marine Species. World Wide Web electronic publication. www.marinespecies.org. 